\title{
DETERMINING THE FIXED ACIDS IN BUTTERS AND MARGARINES.
}

Bx Thos. P. Blunt, M.A., F.C.S., F.I.C.

Ir is obviously an advantage in examining " margarines" to determine the insoluble acids ; this is especially the case with mixtures containing a proportion of genuine butter; for, corroborative proof is here of the greatest importance.

I am in the habit of utilising the residue from Reichert's process for this purpose, and have found the method work so well and accurately that $I$ venture to bring the details before the readers of the ANALYST. I use a very slight excess of sulphuric acid in destroying the soap previously to distilling, to obviate charring when the fluid in the flask becomes concentrated, and I add two or three pieces of pumice, according to the usual practice, to prevent bumping. When the distillation is complete I filter the distillate (which is nearly clear with pure butter, but full of white crystals where "margarine" is under examination) and reserve the filter; the flask (about 300 c.c. capacity) 
is now nearly filled with distilled water, boiled, and cooled; this is repeated, and the water poured from the cake of fat: the flask is now reversed on a ring of the retortstand, over the reserved filter, and allowed to drain. The flask and its contents are then heated in the water oven, the clear, fatty acids poured from the pumice into a flat glass of the usual form, the pumice and flask washed two or three times with a warm mixture of equal parts of ether and alcohol, which is passed through the filter into the glass containing the fat. The solvent is then evaporated off, and the acids dried and weighed as usual.

There is little new in the above, but it is a decided saving of labour over a second saponification, and the results are exact; a genuine butter gave a distillate requiring 13.6 c.c. of decinormal soda for saturation, and fixed acids 87.9 per cent. One obvious advantage of the modification is the removal of the greater part of the volatile acids by distillation, and the comparatively slight washing of the fixed acids required. 